\title{
ON THE FRATTINI SUBGROUP \\ OF A RESIDUALLY FINITE GENERALIZED FREE PRODUCT
}

\author{
R. B. J. T. ALLENBY AND C. Y. TANG ${ }^{1}$
}

ABSTRACT. Let $G=(A * B)_{H}$ be the generalized free product of the groups $A, B$ amalgamating the subgroup $H$, and let $\Phi(G)$ denote its Frattini subgroup. In support of the conjecture that $\Phi(G) \subseteq H$. whenever $G$ is resiually finite and $H$ satisfies a nontrivial identical relation, we show, amongst several other things, that the above inequality is indeed valid if in addition at least one of the following holds: (i) $A, B$, each satisfies a nontrivial identical relation; (ii) $G$ is finitely generated; (iii) $H$ is nilpotent. In particular (i) completes earlier investigations of the second author. The methods of proof are, however, different.

1. The questions concerning the Frattini subgroup of a generalized free product of groups, first raised by Higman and Neumann [6] have, so far, been answered only for certain special classes of groups. In [7, Theorem 3.5] the second author proved the following result (throughout we assume $A \neq H$ $\neq B$ ): If $G=(A * B)_{H}$ is residually finite (in symbols, $G \in R \mathcal{F}$ ) then $\Phi(G)$ is contained in $H$ provided one of the following conditions is satisfied:

(i) $A, B$ each satisfies a nontrivial identical relation which is not satisfied by the infinite dihedral group; (ii) $A, B$ each satisfies a nontrivial identical relation and both $|A: H|$ and $|B: H|$ are greater than 2 ; (iii) $|A: H|=|B: H|=2$.

In this paper we improve this result to read:

Theorem 1. Let $G=(A * B)_{H} \in R \mathcal{F}$. If $H \varsubsetneqq C \subseteq A$ and $H \varsubsetneqq D \subseteq B$, where $C, D$ each satisfies a nontrivial identical relation, then $\Phi(G) \subseteq H$.

It is more convenient to begin with a proof of our main result, namely:

Theorem 2. Let $G=(A * B)_{H} \in R \mathcal{F}$. If $H$ satisfies a nontrivial identical relation and if $G$ is finitely generated then $\Phi(G) \subseteq H$.

Received by the editors October 30, 1973.

AMS (MOS) subject classifications (1970). Primary 20E20, 20E30; Secondary $20 \mathrm{E} 10,20 \mathrm{~F} 30$.

Key words and phrases. Frattini subgroup, residually finite, generalized free product, identical relation.

1 The work of the second author was partly supported by a grant from Canadian National Research Council No. A-4064. 
The proof of Theorem 2 leads naturally to

Theorem 3. Let $G=(A * B)_{H} \in R \mathcal{F}$. If $H$ satisfies a nontrivial identical relation and if $A, B$ possess proper subgroups $K, L$ of finite index containing $H$, then $\Phi(G) \subseteq H$.

This in turn leads, on closer investigation, to a proof of Theorem 1 and also to

Theorem 4. Let $G=(A * B)_{H} \in R \mathcal{F}$. If neither $A$ nor $B$ lies in the variety generated by $H$ then $\Phi(G) \subseteq H$.

Finally a result of Djokovic and Tang [3] helps us prove

Theorem 5. Let $G=(A * B) \in R \mathcal{F}$. If $H$ is nilpotent then $\Phi(G) \subseteq H$.

2. In what follows we shall need the following result from [1].

Theorem A. Let $G=(A * B)_{H}$. If $G$ contains a nontrivial normal subgroup $N$ such that $N \cap H=1$ then $\Phi(G) \subseteq H$.

As an important consequence of this and work of Dyer [4] we have

Theorem 0. Let $G=(A * B)_{H}$. Suppose $X \triangleleft A, Y \triangleleft B$ are finite and such that $X \cap H=Y \cap H$ and not both of $X, Y$ are contained in $H$. Then $\Phi(G) \subseteq H$.

Proof. From the proof of Dyer's Proposition 1 [4, p. 133] we see that the normal closure $\langle X, Y\rangle^{G}$ of $\langle X, Y\rangle$ in $G$ is an extension of a free group by a finite group of order $n$, say. Since at least one of $X, Y$ is not in $H$ the free group is seen to be nontrivial and consequently $\langle X ; Y\rangle^{G}$ contains a nontrivial characteristic free subgroup (namely that generated by the $n$th powers of its elements). This subgroup is clearly normal in $G$ and intersects $H$ trivially whence $\Phi(\bar{G}) \subseteq H$ by Theorem $A$.

We can now give the

Proof of Theorem 2. First suppose $\Phi(G) \cap A=\Phi(G) \cap B=\Phi(G) \cap$ $H=T$, say. Then $T \varangle G$. Passing to $G / T=(A / T * B / T)_{H / T}$ we see that $G / T$ contains the subgroup $\Phi(G) / T=\Phi(G / T)$, a normal subgroup which intersects $H / T$ trivially. It follows immediately from Theorem $A$ that $\Phi(G / T) \subseteq H / T$ and hence that $\Phi(G) \subseteq H$.

Thus we may suppose that the re exists $a \in A$ (say) such that $a \in \Phi(G)$ but $a \notin H$. From this it follows that $|A: H|>2$ : for, if $|A: H|=2$ we may choose $1, a$ as coset representatives of $A$ modulo $H$ and then obtain the 
obvious contradiction $G=\langle A, B\rangle=\langle a, H, B\rangle=\langle a, B\rangle=B$ since $a \in \Phi(G)$, the set of nongenerators of $G$.

Now select $b \in B \backslash H$ arbitrarily and let $a_{1}$ be an element of $A$ not in $H \cup a H$. Clearly $u=a_{1}^{-1}\left(a \cdot b^{-1} a b\right) a_{1}$ is an element of $\Phi(G)$ which, in reduced form, has both its initial and final letter in $A \backslash H$, since $a_{1}^{-1} a \not k H$. Let $S=\left\langle u, b^{-1} u b\right\rangle$. It is easily seen that $S$ is a free group of rank 2 and that $S \subseteq \Phi(G)$. Let $w\left(x_{1}, \cdots, x_{n}\right)=1$ be a nontrivial identical relation satisfied by $H$ and let $y_{1}, \cdots, y_{n}$ be any $n$ elements of $S$ which freely generate a subgroup of rank $n$. Then $w\left(y_{1}, \cdots, y_{n}\right)$ is a nontrivial element of $S$.

Since $G \in R \mathcal{F}$ there exists a normal subgroup $N$ of finite index in $G$ such that $w\left(y_{1}, \cdots, y_{n}\right) \notin N$. Setting $U=N \cap A \cap \Phi(G), V=N \cap B \cap$ $\Phi(G)$ we see that $U \triangleleft A, V \triangleleft B$ and $U \cap H=H \cap V$. Consequently we can form the factor amalgam $(A / U, B / V ; H / U \cap V)$ and its generalized free product $\bar{G}$. Now $\bar{G}$ is a proper free product: from $H U=A$ it follows that $H(\Phi(G) \cap A)=A$ whence $G=\langle A, B\rangle=\langle H, \Phi(G) \cap A, B\rangle=\langle\Phi(G), B\rangle=B$ since $G$ is finitely generated (see, for example, [2]), an obvious contradiction. If $\psi$ denotes the natural map from $G$ to $\bar{G}$ we see that $\operatorname{ker} \psi \subseteq N \cap$ $\Phi(G)$. Further $a \in A \cap \Phi(G)$. However $a \not k \operatorname{ker} \psi$ since $w\left(y_{1}, \cdots, y_{n}\right)$ and consequently $a$ is not in $N$. Thus $\bar{A}=A / U$ contains the nontrivial finite normal subgroup $\bar{A} \cap \Phi(\bar{G}) \cong A \cap \Phi(G) / U, \bar{B}=B / V$ contains the finite normal subgroup $\bar{B} \cap \Phi(\bar{G}) \cong B \cap \Phi(G) / V$ and the intersections of these subgroups with $\bar{H}$ are equal. Thus either $\bar{A} \cap \Phi(\bar{G})=\bar{B} \cap \Phi(\bar{G})$ in wh ich case $\Phi(\bar{G}) \subseteq \bar{H}$ as at the beginning of this proof, or at least one of $\bar{A} \cap$ $\Phi(\bar{G}), \bar{B} \cap \Phi(\bar{G})$ properly contains $\bar{H} \cap \Phi(\bar{G})$ so that Theorem 0 is applicable to show again that $\Phi(\bar{G}) \subseteq \bar{H}$.

Thus $a$ maps under $\psi$ onto $a \psi \in \Phi(\bar{G}) \subseteq \bar{H}$. Hence $w\left(y_{1}, \cdots, y_{n}\right) \psi \in \bar{H}$ : further each $y_{i} \psi \in \Phi(\bar{G}) \subseteq \bar{H}$, hence $w\left(y_{1}, \cdots, y_{n}\right) \psi=1$ in $\bar{H}$, i.e. $w\left(y_{1}, \cdots, y_{n}\right) \in \operatorname{ker} \psi \subseteq N$, a contradiction. Thus Theorem 2 is proved.

Another condition which ensures $H U \neq A$ and $H V \neq B$ as required in the proof of Theorem 2 is the existence in $A, B$ of proper subgroups of finite index which contain $H$. Indeed, if $H \subseteq K \varsubsetneqq A$ and $|A: K|<\infty$, then $H U \neq A$. For otherwise, $K(\Phi(G) \cap A) \supseteq H U=A$ and consequently $A$ is generated by $K$ and finitely many elements $\phi_{1}, \cdots, \phi_{t}$ of $\Phi(G)$. This leads to $G=$ $\langle A, B\rangle=\left\langle\phi_{1}, \cdots, \phi_{t}, K, B\right\rangle=\langle K, B\rangle=(K * B)_{H} \varsubsetneqq G$, a contradiction. Thus we have proved Theorem 3 .

To prove Theorem 4 we note that $H$ satisfies a nontrivial identical re- 
lation; for, if not, $H$ generates the variety of all groups, contradicting the fact that $A$ and $B$ do not lie in this variety. Now $G \in R \mathcal{F}, G$ possesses a collection $N_{\lambda}(\lambda \in \Lambda)$ of normal subgroups of finite index such that $\bigcap_{\boldsymbol{\lambda} \in \mathbf{\Lambda}} N_{\lambda}=1$. Theorem 3 shows that if there exist $\mu, \nu \in \Lambda$ such that $H\left(A \cap N_{\mu}\right) \neq A$ and $H\left(B \cap N_{\nu}\right) \neq B$ (since $H$ satisfies a nontrivial identical relation) then $\Phi(G) \subseteq H$. At the other extreme if $H\left(A \cap N_{\lambda}\right)=A$ for all $\lambda \in \Lambda$ and if $H\left(B \cap N_{\lambda}\right)=B$ for all $\lambda \in \Lambda$, then again $\Phi(G) \subseteq H$. For, then, choosing $N=N_{\lambda} \triangleleft G$ and of finite index as before so that $w\left(y_{1}, \cdots, y_{n}\right) \not N$ we see that $a N \in H N / N, y_{i} N \in H N / N$ and $w\left(y_{1}, \cdots, y_{n}\right) N=$ $w\left(y_{1} N, \cdots, y_{n} N\right)=1$ in $H N / N$, i.e. $w\left(y_{1}, \cdots, y_{n}\right) \in N$, a contradiction. Thus if $G$ is to be such that $\Phi(G) \underline{\not} H$ we must have either $H\left(A \cap N_{\lambda}\right)=A$ for all $\lambda \in \Lambda$ or $H\left(B \cap N_{\lambda}\right)=B$ for all $\lambda \in \Lambda$, but not both. It then follows that either $A$ or $B$ must satisfy the same identical relations as $H$. This proves Theorem 4.

To prove Theorem 1 we suppose, as before, that there exists $a \in A \cap$ $\Phi(G)$ such that $a \notin k H$. By the remarks above we may also suppose that either (i) $H\left(A \cap N_{\lambda}\right)=A$ for all $\lambda \in \Lambda$ or (ii) $H\left(B \cap N_{\lambda}\right)=B$ for all $\lambda \in \Lambda$, but not both. Suppose (ii) holds. Then there exists $\mu \in \lambda$ such that $H\left(A \cap N_{\mu}\right) \neq A$. Consequently $H\left(A \cap \Phi(G) \cap N_{\mu} \cap N_{\nu}\right) \neq A$ for each $\nu \in \Lambda$. Thus if $\Phi(G) \cap B=\Phi(G) \cap H$ the factor amalgam $(A / U, B / V ; H / U \cap V)$, where $U=A \cap \Phi(G) \cap N_{\mu} \cap N_{\nu}, V=B \cap \Phi(G) \cap N_{\nu} \cap N_{\mu}$, is a proper amalgam for all $\nu \in \Lambda$ and we can proceed as before. Hence if (ii) holds and $\Phi(G) \underline{\not} H$ there must exist $b \in B \cap \Phi(G)$ such that $b \not \notin H$. Therefore we may assume, without loss of generality, that (i) holds. Consider the generalized free product $(A * D)_{H}$. It is residually finite and it follows (because of (i)) from Theorem 2 of Gregorac [5] that $H$ has index either 1 or 2 in $\bigcap_{\lambda} H\left(A \cap N_{\lambda}\right)$; that is, $H$ is of index 1 or 2 in $A$. Both of these possibilities are, however, prohibited; the first trivially and the second by the remarks in the second paragraph of the proof of Theorem 2. This proves Theorem 1 .

Next we give the

Proof of Theorem 5. By the remarks above we may assume that (i) there exists $a \in A \backslash H$ such that $a \in \Phi(G)$; (ii) $H\left(A \cap N_{\lambda}\right)=A$ for all $\lambda \in \Lambda$. Then $(\alpha) A$ is nilpotent (of the same class as $H$ ) so that $H^{A} \neq A$ and $(\beta)$ there exists $\mu \in \Lambda$ such that $H\left(B \cap N_{\mu}\right) \neq B$. As usual we now select $\nu \in \Lambda$ such that $w\left(y_{1}, \cdots, y_{n}\right) \notin N_{\nu}$. Set $N=N_{\nu} \cap N_{\mu}$. Now look at the (obviously proper) generalized free product $\bar{G}$ on the amalgam $\left(A / N \cap H^{A}, B / N \cap B ; H / H \cap N\right)$. Let $\psi$ denote the natural homomorphism 
from $G$ onto $\bar{G}$. Then $a \psi \in \Phi(\bar{G})$. But $\Phi(\bar{G}) \subseteq H / H \cap N$ by Theorem 1 of [3] since $H / H \cap N$ is a finite group. As before it follows that $w\left(y_{1}, \cdots, y_{n}\right) \in$ ker $\psi \subseteq N-a$ contradiction.

Finally we state formally the conjecture: If $G=(A * B)_{H} \in R \mathcal{F}$ and if $H$ satisfies a nontrivial identical relation, then $\Phi(G) \subseteq H$.

\section{REFERENCES}

1. R. B. J. T. Allenby and C. Y. Tang, On the Frattini subgroups of generalized free products, Bull. Amer. Math. Soc. 80 (1974), 119-121.

2. John D. Dixon, Problems in group theory, Blaisdell, Waltham, Mass., 1967. MR $36 \# 1514$.

3. D. Ž. Djoković and C. Y. Tang, On the Frattini subgroup of generalized free products with amalgamations, Proc. Amer. Math. Soc. 32 (1972), 21-23.

4. J. L. Dyer, On the residual finiteness of generalized free products, Trans. Amer. Math. Soc. 133 (1968), 131-143. MR 38 \#5930.

5. R. J. Gregorac, On residually finite generalized free products, Proc. Amer. Math. Soc. 24 (1970), 553-555. MR 41 \#5498.

6. G. Higman and B. H. Neumann, $O_{n}$ two questions of Itô, J. London Math. Soc. 29 (1954), 84-88. MR 15, 286.

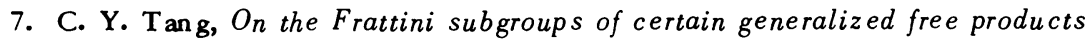
of groups, Proc. Amer. Math. Soc. 37 (1973), 63-68. MR $46 \# 9176$.

SCHOOL OF MATHEMATICS, UNIVERSITY OF LEEDS, LEEDS, ENGLAND

DEPARTMENT OF PURE MATHEMATICS, UNIVERSITY OF WATERLOO, WATERLOO, ONTARIO, CANADA 